\title{
Imagen de Mario Bunge
}

Gustavo E. Romero ${ }^{\dagger}$

Pablo M. Jacovkis ${ }^{*}$

El siglo XVII no sólo fue el de la consolidación de la revolución científica iniciada en el siglo anterior por Nicolás Copérnico, Tycho Brahe y Johannes Kepler, entre otros, sino que además fue el siglo de los grandes sistemas filosóficos acordes a los avances de la ciencia. Descartes, Leibniz y Spinoza desarrollaron vastos programas filosóficos que contemplaban todos los aspectos del pensamiento filosófico, incluyendo la metafísica, la teoría del conocimiento, la ética e incluso la estética. Una característica de estos sistemas es que estaban informados por la ciencia de la época, con la que eran compatibles. Esta tendencia se acentuó en el siglo XVIII con la Ilustración y el movimiento enciclopédico. Quizás la manifestación más acabada de un sistema integral de filosofía científica en este período fue el Système de la Nature (Sistema de la Naturaleza), publicado en 1770 por Paul Henri Thiry, barón de Holbach. Con la Revolución Francesa y luego el advenimiento del Romanticismo surge una contra-Ilustración que terminaría llevando al Idealismo alemán de Fichte y Hegel, y luego desembocaría en los irracionalismos del siglo XX. Como reacción a la filosofía extremadamente oscura del Idealismo, se produce el nacimiento de una filosofía científica de naturaleza crítica y enemiga de la especulación metafísica. El Positivismo Lógico primero y luego la filosofía analítica anglosajona dominaron la academia durante buena parte del siglo XX. Sobre este panorama de fragmentación y dedicación al análisis de la filosofía académica en el siglo pasado se destacan las figuras de dos pensadores que han logrado una obra de unidad notable y enorme alcance: Bertrand Russell y Mario Bunge. En Bunge, en particular, el sistema filosófico ha resurgido como una propuesta que, al igual que en los grandes ejemplos de la Antigüedad, abarca desde la semántica hasta la ética, pasando por la ontología y la epistemología. Bunge es hoy, en pleno siglo XXI, una rara avis filosófica: autor de un sistema de filosofía materialista forjado a la luz de los más recientes avances de la ciencia contemporánea, se ha dedicado incansablemente a resolver problemas filosóficos en forma directa y clara, con propuestas no sólo audaces e innovadoras, sino también contrastables a la luz del cuerpo total de nuestro conocimiento científico.

Mario Bunge nació en Florida Oeste, a 17 km de la ciudad de Buenos Aires, Argentina, el 21 de septiembre de 1919. Hijo de un médico y diputado socialista de familia patricia, el Dr. Augusto Bunge, el joven Mario vivió siempre en un ambiente de libertad intelectual y compromiso social. Su madre, de origen alemán, era Marie Müser, quien había sido enfermera de la Cruz Roja en China. El joven Mario pronto se sintió atraído por la filosofía. En 1936 comenzó a realizar lecturas más o menos sistemáticas de tópicos filosóficos, pero pronto se convenció de que si quería hacer filosofía seriamente debía primero conocer a fondo la ciencia. Se inscribió en la carrera de física de la Universidad Nacional de La Plata en 1938, donde fue estudiante hasta 1944; más tarde se doctoró en 1952 con una tesis sobre la cinemática del electrón relativista. Su mentor de física fue Guido Beck (1903-1988), quien había sido asistente de Heisenberg en Leipzig. En 1944 Bunge publicó su primer artículo de física, en la revista Nature, sobre las secciones eficaces de las interacciones nucleares. En ese mismo año participó de la fundación de la Asociación Física Argentina. Pero ya antes Bunge había incursionado en la filosofía, publicando en 1939 un trabajo titulado "Introducción al estudio de los grandes pensadores". En 1944

\footnotetext{
† Instituto Argentino de Radioastronomía (IAR, CONICET), C.C. No. 5, 1894 Villa Elisa, Buenos Aires, Argentina. Para contactar al autor, por favor, escribir a: romero@fcaglp.unlp.edu.ar.

* Universidad Nacional de Tres de Febrero y Universidad de Buenos Aires, Argentina. Para contactar al autor, por favor, escribir a: pjacovkis@untref.edu.ar

Metatheoria 7(2)(2017): 3-16 ISSN 1853-2322.

(c) Editorial de la Universidad Nacional de Tres de Febrero. Publicado en la República Argentina.
} 
fundó la revista de filosofía Minerva, donde se publicaron artículos de varios de los pensadores más prestigiosos de Latinoamérica. El propio Bunge escribió varios artículos para la revista, que duró un año. Uno de esos artículos, “Qué es la epistemología?", es quizás el primer artículo de filosofía de la ciencia escrito en castellano.

En 1952 Bunge publicó su primer artículo en inglés, "What is Chance?", donde proponía una interpretación objetiva del cálculo de probabilidades y afirmaba que lo que a un nivel ontológico era azar, podía ser causalidad en otro. Durante la segunda mitad de los años 1950 Bunge, ahora profesor de física en la Universidad de La Plata y de física y filosofía en la de Buenos Aires, continuó publicando artículos de temas diversos de epistemología y ontología en revistas de habla inglesa. En 1959 aparece su libro Causality, publicado por Harvard University Press y re-editado en forma revisada por Dover en 1979. En 1963 deja Argentina definitivamente y viaja primero a Estados Unidos y luego a Alemania con una beca Humboldt. En Freiburg completa su impresionante Foundations of Physics, libro riguroso y singular, aún hoy no superado en su especialidad. En el capítulo 2 de esa obra feliz Bunge traza un programa de filosofía científica al que luego se dedicará en forma exhaustiva por casi 20 años. Foundations of Physics ve la luz en 1967. Ese mismo año aparece su tratado Scientific Research, en dos volúmenes. Ese libro, editado luego en castellano, se transformaría en un clásico en el mundo hispanoparlante.

A comienzos de los años 1970, ya establecido como profesor en McGill University en Montreal, Canadá (a donde llegó en 1966), Bunge se impone desarrollar un sistema completo de filosofía que incluya desde la semántica hasta la ética. Se propone construir una filosofía científica, sistémica, materialista y realista, y se aboca a resolver problemas filosóficos concretos con herramientas formales y exactas. El resultado fue el monumental Treatise of Basic Philosophy, publicado entre 1974 y 1989 por Reidel (ahora Kluwer) en 8 tomos y 9 volúmenes. En este siglo esta obra está siendo traducida al castellano por Rafael González del Solar para la editorial española Gedisa. El Treatise es uno de los grandes hitos filosóficos del siglo XX. Desde la Ilustración que no se veía en Occidente un emprendimiento filosófico de tal envergadura. Bunge luego completaría y actualizaría esta obra asombrosa con una serie de libros complementarios publicados en la primera década del nuevo siglo. Los más importantes de éstos son Emergence and Convergence, Chasing Reality y Mind and Matter.

Luego de completar su tratado, Bunge se dedicó durante más de 10 años al estudio de los fundamentos y las implicaciones filosóficas de las ciencias sociales. Entre sus libros en este tema, se destacan Finding Philosophy in Social Sciences y Social Science under Debate, libros polémicos y estimulantes. Publicó, asimismo, una gran cantidad de artículos sobre ciencias sociales durante esa década.

Bunge no sólo ha descollado en producir vastos análisis de grande áreas de la filosofía, sino que ha sido un incansable filósofo de ciencias especiales, habiendo publicado extensamente sobre filosofía de la física, de la mente, de la psicología, de la economía, de las matemáticas, así como sobre pseudociencia e ideología.

En años recientes ha incursionado en filosofía práctica y política con sus obras Medical Philosophy y Political Philosophy. Actualmente, a los 97 años, se encuentra trabajando sobre aspectos filosóficos de la práctica científica.

A lo largo de más de 70 años de actividad académica Mario Bunge ha producido una obra de más de 80 volúmenes y cientos de artículos que no tiene precedentes en el panorama filosófico contemporáneo. Ningún otro filósofo de origen hispánico ha tenido una repercusión similar a la suya. Y sin embargo, en el mundo anglosajón su obra es relativamente ignorada. La razón, nos atrevemos a conjeturar, es doble. Por un lado, Bunge es una personalidad polémica que no se calla las críticas, lo cual muchas veces es recibido con desagrado en ámbitos académicos no habituados al debate científico. Por otro, la amplitud y profundidad del trabajo de Bunge, así como su tendencia a tratar de problemas filosóficos sin preocuparse por las modas pasajeras, hacen que su obra no sea del paladar de los filósofos analíticos contemporáneos, más proclives a la ultra-especialización y a ignorar la ciencia en función de los juegos lógicos y semánticos. 
Es posible que una completa apreciación de la obra de Mario Bunge deba esperar aún mucho tiempo. Su trabajo no es para el lector casual o para quien teme adentrarse en terrenos difíciles. Tampoco para quien, preocupado por los temas de moda, evita plantearse preguntas fundamentales. Sin embargo, como todo lo que es compatible con la ciencia, su obra terminará imponiéndose. No es de extrañar que sean precisamente los científicos los primeros en reconocer su extraordinaria importancia. Una gran edición crítica de sus obras es aún una terea esencial...y pendiente.

Podríamos concluir aquí esta reseña de la extraordinaria creatividad, importancia y rigor de Mario Bunge como filósofo, en la que su paso previo por la ciencia tuvo un papel esencial (¡y qué papel!: no abundan en el mundo los estudiantes de doctorado residentes en un país lejano y sin tradición científica que publican un artículo en Nature o Physical Review, y como ¡único autor!) y habríamos dejado en claro por qué se destaca tanto. Pero vale la pena analizar el contexto argentino en el cual se formó, y en cual participa pese a que hace más de cincuenta años que no reside en el país, para describir no sólo la extraordinaria fuerza de voluntad que llevó a un autodidacta en filosofía a ser conocido y respetado en el mundo académico internacional antes de radicarse en el primer mundo, sino también el compromiso que siempre tuvo con su época y su sociedad.

En 1938, siendo un flamante estudiante de física en la Universidad de La Plata, Bunge creó la Universidad Obrera Argentina (OUA), que daba clases a obreros sindicalizados. Independientemente de que, en algún sentido, la UOA puede considerarse un antecedente de la Universidad Obrera Nacional, institución oficial creada durante el gobierno de Perón con el objeto de formar ingenieros surgidos de la clase obrera -que subsiste, con el nombre de Universidad Tecnológica Nacional, pero convertida en una universidad "normal", diferenciada de las otras universidades nacionales solamente por el hecho de que su estructura administrativa es distinta, con facultades distribuidas por todo el país- el esfuerzo de Bunge, ayudado por algunos amigos, de dar clases a nivel universitario a obreros (y de llevar adelante toda la gestión administrativa y contable, además de afrontar personalmente erogaciones no desdeñables) fue realmente notable. A pesar de la desconfianza y vigilancia policial (en esa época Bunge era un marxista confeso) en la UOA dieron clases personalidades relevantes (o de futura relevancia) como Arturo Frondizi, que llegó a Presidente de la Nación, y Juan Atilio Bramuglia, que fue Ministro de Relaciones Exteriores durante la primera presidencia de Perón. Bunge renunció a la dirección de la UOA en agosto de 1943, debido a la muerte de su padre, y la UOA lo sobrevivió muy poco tiempo: a fin de ese año la dictadura militar que había destituido al presidente conservador (fraudulento) Ramón Castillo en junio de 1943 disolvió la UOA y la policía allanó su local y se robó todo, como lo relata en sus memorias (Bunge, 2014b). La UOA tenía en esa época más de mil alumnos, lo cual indica el éxito que había obtenido. Y todo esto siendo Bunge simultáneamente estudiante universitario y debiendo mantener una familia (ya estaba casado y tenía dos hijos pequeños cuando cerró la OUA).

Estudiante de grado y luego de doctorado, trabajando de lo que pudiere (con muchas puertas cerradas, pues ya estaba fichado como comunista por la dictadura militar) se las arregló también para editar y administrar la ya mencionada revista de filosofía Minerva, para la cual además escribió varios artículos. Minerva llegó a publicar seis números (Horacio Tarcus logró recuperar la colección, que está archivada en el Centro de Documentación e Investigación de la Cultura de Izquierdas en Argentina, CEDINCI; es posible que haya otra colección en algún archivo de la Policía Federal). Pero al término de la agotadora aventura de Minerva, ganándose la vida esencialmente dando clases particulares (con un cargo de auxiliar docente en física en Buenos Aires desde septiembre de 1948 hasta marzo de 1953, en que su contrato no fue renovado debido -al menos así lo supone él- a que se negó a que le descontaran el 10\% de su sueldo para la Fundación Eva Perón o a que no se afilió al Partido Peronista), sumamente aislado (al disentir con el Partido Comunista muchos de sus militantes también le hicieron el vacío), con un director de tesis radicado en Córdoba -y en esa época no había Internet para tener comunicación instantánea- e incluso con un corto período detenido por la policía y una breve interrupción de su relación estudiante-director con Beck, felizmente superada, pudo defender su tesis de doctorado, lo cual, como él comenta en sus memorias, al principio no le sirvió para conseguir trabajo, al no gozar de los favores del gobierno. Sobrevivió mediante alumnos particulares, alguna beca 
y estadías cortas en Brasil, Bolivia y Chile. Y fue justamente durante el gobierno de Perón, antes de su derrocamiento en 1955, cuando Bunge formó parte de uno de esos típicos grupos de estudio que se armaban entre intelectuales que tenían el acceso vedado a la universidad; en este caso, el grupo (el Círculo Filosófico de Buenos Aires) fue particularmente brillante e interdisciplinario: sus integrantes eran, aparte del propio Bunge, el matemático Manuel Sadosky, figura señera y uno de los "padres fundadores" de la informática en Argentina; Gregorio Weinberg, hombre de la cultura y humanista; Hernán Rodríguez Campoamor, quien tradujo al castellano varios libros de Bunge; Federico "Pipo" Westerkamp, físico; Hersch "Coco" Gerschenfeld, neurobiólogo de nivel internacional; y Enrique Mathov, médico. Bunge indica en el prólogo de Causality que el contenido de dicho libro había sido discutido en 1954 por los entonces integrantes del Círculo (todos los mencionados, con excepción de Gerschenfeld), lo cual da una idea de la calidad de las discusiones (y de los discutidores). De los participantes mencionados, Bunge terminó radicado en Montreal, Rodríguez Campoamor en Nueva York y Ginebra, y Gerschenfeld en París; esas emigraciones son una pequeña muestra del costo intelectual para Argentina de sus avatares políticos.

A la caída de Perón, derrocado por un golpe militar en 1955 en el contexto de una sociedad terriblemente polarizada entre sus partidarios y sus enemigos, se produjo un hecho único en la larga y lamentable historia de los golpes militares en Argentina: dado que la mayor parte de los estudiantes universitarios, y numerosos intelectuales y académicos, eran manifiestamente antiperonistas, las nuevas autoridades universitarias integraron las cátedras de las universidades con prestigiosos académicos y jóvenes intelectuales que, o bien habían sido echados de la universidad durante el gobierno anterior, o bien directamente nunca habían podido acceder a cargos para los cuales estaban perfectamente capacitados. Entre ellos, por supuesto, Mario Bunge. De alguna manera Bunge repartió al principio su actividad académica en la Universidad de Buenos Aires entre sus "dos amores": la física y la filosofía, mediante cargos de profesor en la Facultad de Ciencias Exactas y Naturales y la Facultad de Filosofía y Letras, respectivamente. En 1958 decidió priorizar uno de los dos amores, el de la filosofía, y se quedó con un cargo de profesor con dedicación exclusiva en Filosofía y Letras. Su estilo era excesivamente frontal para la cultura imperante en dicha facultad en el área de filosofía; además, pocos meses después del derrocamiento mediante un golpe militar del presidente Arturo Frondizi se produjo un sangriento enfrentamiento entre dos fracciones de las Fuerzas Armadas, que eran los verdaderos dueños del poder en el país, el cual gobernaban a través del presidente interino José María Guido, que dependía absolutamente de ellas (además, el Congreso había sido disuelto y las provincias intervenidas). Tanto la situación profesional de Bunge como la situación política del país lo llevaron a tomar la decisión de radicarse en el exterior con Marta Cavallo, su segunda esposa, a principios de 1963; y desde 1966 residen ininterrumpidamente, como ya se mencionó (salvo un breve período en México), en Montreal, donde actualmente ambos son profesores eméritos, Mario de filosofía y Marta de matemáticas, en McGill University.

Sin embargo, si bien Bunge vive fuera de Argentina desde hace más de medio siglo, nunca se desentendió de los problemas de su país, y sus opiniones e intervenciones escritas y orales, siempre polémicas, tanto sobre política como sobre temas académicos, son un aporte enriquecedor en las áreas en las cuales se insertan. En algunos casos, sus opiniones, por escrito o a través de reportajes, se circunscriben a la Argentina, como en su artículo de Ciencia Nueva (Bunge, 1972a), que provocó fuertes discusiones (Ciencia Nueva fue durante el período en que se publicó -1970-1974- una muy interesante y valiosa fuente de discusiones sobre ciencia y política, en particular de Argentina), y en otros casos se refiere a la actualidad en general, en particular la realidad latinoamericana, como en su valioso artículo sobre la investigación científica en los países en desarrollo (Bunge, 1968c) reproducido después parcialmente en una compilación de Jorge Sabato.

En los últimos años, con una vitalidad extraordinaria, Bunge comenzó ¡a los noventa años! a coordinar (y participar activamente en) un seminario de filosofía de la ciencia en la Facultad de Ciencias Exactas y Naturales de la Universidad de Buenos Aires, seminario que se ofreció ininterrumpidamente durante un mes entre 2010 y 2015, con un éxito notable. Es decir, con Bunge pasando un mes en Buenos Aires cada uno de esos años como profesor visitante de dicha facultad. Es 
realmente admirable no sólo verlo exponer, sino instando a los asistentes a que le hagan preguntas, a fin de contestarlas, rebatirlas o aceptarlas.

Por último, como puede observarse perfectamente en la bibliografía de sus obras indicada más abajo, dos características de Bunge llaman la atención: por un lado, casi toda su obra es producto individual de él: hay muy pocos trabajos en colaboración, y cuando los hay, en general, no son sobre los temas académicos en los cuales está interesado sino sobre temas políticos o más generales (sobre los cuales, por supuesto, también está interesado). Y, por otro lado, que desde muy joven decidió "jugar en primera": recordemos las ya mencionadas contribuciones a Nature y a Physical Review. En los años 1950 vendrían The Americal Journal of Physics, The Bristish Journal for the Philosophy of Science y muchos más. Bunge no solamente tenía desde muy joven ideas propias originales, sino que era plenamente consciente de su valor y, a diferencia de muchos científicos de países subdesarrollados que no se animan a enviar sus trabajos a revistas importantes internacionales sin el apoyo y la animación de alguna figura muy respetada, estaba dispuesto desde temprano a competir en la arena internacional. Y lo hizo. Y triunfó.

\section{Bibliografía básica de Mario Bunge}

Bunge, M. (1939), "Introducción al estudio de los grandes pensadores", Conferencias (Buenos Aires), III, pp. 105-109 y 124-126.

- (1943a), El tricentenario de Newton, Buenos Aires: Universidad Obrera Argentina.

- (1943b), Significado físico e histórico de las ecuaciones de Maxwell, Buenos Aires: Universidad Obrera Argentina.

- (1943c), "La epistemología positivista”, Nosotros (Buenos Aires) 8(93): 283-290.

- (1944a), "Una nueva representación de los tipos de fuerzas nucleares", Revista de la Facultad de Ciencias Físicomatemáticas (La Plata) 3: 221-239.

- (1944b), "A New Representation of Types of Nuclear Forces", Physical Review 65: 249.

- (1944c), “QQué es la epistemología?", Minerva 1: 27-43.

- (1944d), "Precursores, predecesores y predictores", Minerva 1: 61-62.

- (1944e), "Una de las posibles metafísicas", Minerva 1: 167-168.

- (1944f), "Auge y fracaso de la filosofía de la naturaleza”, Minerva 1: 213-235.

- (1944g), "Una nueva interpretación de Rousseau", Minerva 1: 274-278.

- (1944h), "Nietsche y la ciencia", Minerva 2: 44-50.

- (1945a), "Neutron-proton Scattering at 8.8 and 13 MeV", Nature 156: 301.

- (1945b), "El spin total de un sistema de más de dos partículas", Revista de la Unión Matemática Argentina 10: 13-14.

- (1945c), “Cómo veía el mundo Florentino Ameghino”, Minerva 2: 184-185.

- (1945d), "Fenómenos de resonancia en la difusión de neutrones por protones", Revista de la Unión Matemática Argentina 11: 35.

- (1951a), "What is Chance?", Science and Society 15: 209-231.

- (1951b), "La fenomenología y la ciencia”, Cuadernos Americanos 4: 108-122.

- (1951c), "Bemerkung über den Massendefekt des Wasserstoffatoms", Acta Physica Austriaca 5: 77-79.

- (1951d), "Mach y la teoría atómica", Boletín del Químico Peruano 3(16): 12-17.

- (1954), "New Dialogues between Hylas and Philonous", Philosophy and Phenomenological Research 15: 192-199.

- (1955a), "A Picture of the Electron”, Nuovo Cimento 6(1): 977-985.

- (1955b), "Strife about Complementarity", British Journal for the Philosophy of Science 6: 1-12 y 141-154. 
- (1955c), La edad del universo, La Paz: Laboratorio de Física Cósmica. Reproducido en Revista Boliviana de Física 8 (2002): 83-101.

- (1955d), "Exposición y crítica del principio de complementariedad", Notas del Curso Interamericano de Física Moderna, La Paz: Laboratorio de Física Cósmica, pp. 27-36.

- (1956a), “Do Computers Think?”, British Journal for the Philosophy of Science 7: 139-148 y 212-219.

- (1956b), “A Survey of the Interpretations of Quantum Mechanics”, American Journal of Physics 24: 272-286.

- (1956c), "A Critique of the Frequentist Theory of Probability", Congresso Internacional de Filosofia, Vol. III, San Pablo: Instituto Brasileiro de Filosofía, pp. 787-792.

- (1956d), "La interpretación causal de la mecánica ondulatoria”, Ciencia e Investigación 12: 448-457.

- (1956e), "Nuevas constantes del movimiento del electrón”, Revista de la Unión Matemática Argentina y de la Asociación Física Argentina 18: 25.

- (1956f), "La antimetafísica del empirismo lógico", Anales de la Universidad de Chile 102: 43-50.

- (1956g), “Beitrag zur Diskussion über philosophische Fragen der modernen Physik”, Deutsche Zeitschrift für Philosophie 4: 467-496.

- (1956h), “iHa progresado la filosofía en el siglo XX?”, Revista do Livro (Río de Janeiro) 1(3/4): 15-21. Existe traducción al portugués: “A filosofía tem progredido durante o século XX?", Revista Filosófica (Coimbra) 8(22): 54 62 (1959).

- (1956i), "Ubicación de la física teórica”, Revista de la Universidad de Buenos Aires 1: 405-409.

- (1957a), "Lagrangian Formulation and Mechanical Interpretation”, American Journal of Physics 25: 211-218.

- (1957b), "Las ideas fundamentales de la mecánica ondulatoria”, Ciencia y Técnica 123(616): 3-21.

- (1957c), "Filosofar científicamente y encarar la ciencia filosóficamente”, Ciencia e Investigación 13: 244.

- (1958a), "Sobre la imagen física de las partículas de spin entero", Ciencia e Investigación 14: 311-315.

- (1958b), "On Multi-dimensional Time”, British Journal for the Philosophy of Science 9(33): 39.

- (1958c), ¿Qué significa 'ley científica’?, México: Universidad Nacional Autónoma de México.

- (1959a), "Reseña de The Logic of Scientific Discovery”, Ciencia e Investigación 15: 216.

- (1959b), Causality: The Place of the causal Principles in Modern Science, Cambridge, MA: Harvard University Press.

- (1959c), Metascientific Queries, Springfiels, IL: Charles C. Thomas.

- (1959d), “QQué es un problema científico?”, Holmbergia 6(15): 47-63.

- (1959e), “Cómo sabemos que existe la atmósfera?”, Revista de la Universidad de Buenos Aires 4(2): 246-260.

- (1960a), "Levels: A semantical Preliminary", Review of Metaphysics 13(3): 396-406.

- (1960b), "The Place of Induction in Science”, Philosophy of Science 27: 262-270.

- (1960c), La ciencia: Su método y su filosofía, Buenos Aires: Siglo Veinte. Reimpresión, Pamplona: Laetoli, 2013.

- (1960d), Cinemática del electrón relativista, Tucumán: Universidad Nacional de Tucumán.

- (1960e), “On the Connections among Levels", Proceedings of the XII ${ }^{\text {th }}$ International Congress of Philosophy 6, Florencia: Sansoni, pp. 63-70.

_ (1960f), "Probabilidad e inducción”, Ciencia y Técnica 129: 262-270.

- (1961a), “Analyticity Redefined”, Mind 278: 239-245.

- (1961b), "Ethics as a Science”, Philosophy and Phenomenological Research 22(2): 139-152.

- (1961c), “Laws of Physical Laws”, American Journal of Physics 29: 518-529.

- (1962a), "Cosmology and Magic”, The Monist 44: 116-141.

- (1962b), “An Analysis of Value”, Mathematicae Notae 18: 95-108.

- (1962c), “The Complexity of Simplicity”, Journal of Philosophy 59(5): 113-135. 
- (1962d), "Causality: A Rejoinder", Philosophy of Science 29(3): 306-317.

- (1962e), "La teoría del conocimiento en nuestro tiempo", Ciencia e Investigación 18: 60-65.

- (1963a), "Tecnología, ciencia y filosofia”, Anales de la Universidad de Chile 121(126): 64-92.

- (1963b), The Myth of Simplicity, Englewood Cliffs, NJ: Prentice-Hall.

- (1963c), "A General Black Box Theory", Philosophy of Science 30(4): 346-358.

- (1964), "Phenomenological Theories", en Bunge, M. (ed.), The Critical Approach, Glencoe, IL: Free Press, pp. 234254.

- (1965), "Physics and Reality", Dialectica 19(3/4): 195-222. Reproducido en Foster, M.H. y M. Martin (eds.) (1966), Probability, Confirmation and Simplicity, Nueva York: Odyssey Press, y en MacKinnon, E.A. (ed.) (1972), The Problem of Scientific Realism, Nueva York: Appleton-Century-Crofts, 1972. Existe traducción al alemán: Physik und Wirklichkeit, en Krüger, L. (ed.) (1970), Erkenntnisprobleme der Naturwissenschaften, Colonia-Berlín: Kiepenheu, pp. 435-457.

- (1966a), "Technology as Applied Science", Technology and Culture 7: 329-347. Reproducido como "Towards a Philosophy of Technology", en Dockx, S. (ed.) (1968), Civilisation, technique et humanisme, Bruselas: Office International de Librairie, pp. 189-210.

- (1966b), "Mach's Critique of Newtonian Mechanics", American Journal of Physics 34: 329-347. Reproducido en Blackmore, J. (ed.) (1992), Ernst Mach. A Deeper Look: Documents and New Perspectives, Berlín-Heidelberg-Nueva York: Springer-Verlag, pp. 243-261. Traducido al alemán como: „Machs Kritik an der Newtonschen Mechanik“, en Kerkhof, F. (ed.) (1967), Symposium aus Anlass des 50. Todestags von Ernst Mach, Freiburg im Bresgau: Ernst Mach Institut, pp. 227-246.

_ (1966c), "On Null Individuals", Journal of Philosophy 63: 776-778.

- (1967a), Foundations of Physics, Berlín-Heidelberg-Nueva York: Springer.

- (1967b), Scientific Research, Berlín-Heidelberg-Nueva York: Springer.

- (1967c), "Physical Axiomatic", Reviews of Modern Physics 39: 463-474.

- (1967d), "Analogy in Quantum Mechanics: From Insight to Nonsense”, British Journal for the Philosophy of Science 18: 265-286.

- (1967e), "The Structure and Content of a Physical Theory", en Bunge, M. (ed.), Delaware Seminar in the Foundations of Physics, Berlín-Heidelberg-Nueva York: Springer, pp. 15-27.

- (1967f), "Quanta and Philosophy", en Proceedings of the 7th Inter-American Congress of Philosophy I, Quebec: Presses de l'Université de Laval, pp. 281-296. Existe traducción al castellano: "Quanta y filosofía", Crítica 1(3) (1967): 41. 64.

- (1968a), "Physical Time: The Objective and Relational Theory", Philosophy of Science 35: 355-388.

- (1968b), "The Maturation of Science”, en Lakatos, I. y A. Musgrave (eds.), Problems in the Philosophy of Science, Ámsterdam: North-Holland, pp. 120-137. Discusiones por L.L. Whyte, K.R. Popper y E.H. Hutton y respuesta del autor: pp. 138-147.

- (1968c), "Filosofía de la investigación científica en los países en desarrollo", Acta Científica Venezolana 19: 118123. Reproducido parcialmente en Sabato, J.A. (comp.) (1975), El pensamiento latinoamericano en la problemática ciencia-tecnología-desarrollo-dependencia, pp. 44-51.

- (1968d), "Scientific Laws and Rules", en Klibansky, R. (ed.), Contemporary Philosophy II, Florencia: La Nuova Italia Editrice, pp. 3-15.

- (1968e), "The Nature of Science", en Klibansky, R. (ed.), Contemporary Philosophy II, Florencia: La Nuova Italia Editrice, pp. 128-140.

- (1968f), "Philosophy and Physics", en Klibansky, R. (ed.), Contemporary Philosophy II, Florencia: La Nuova Italia Editrice, pp. 167-199.

- (1968g), "On Mach's Nonconcept of Mass", American Journal of Physics 36: 167-168.

- (1968h), "Problems and Games in the Current Philosophy of Science", en Proceedings of the XIV ${ }^{\text {th }}$ International 
Congress of Philosophy I, Viena: Herder, pp. 566-574.

- (1968i), "Physique et métaphysique du temps", en Proceedings of the XIV ${ }^{\text {th }}$ International Congress of Philosophy II, Viena: Herder, pp. 623-629.

- (1968j), "Conjunction, Succession, Determination, and Causation", International Journal of Theoretical Physics 1: 299-315. Existe traducción al francés: “Conjonction, succession, determination, causalité”, en J. Piaget (ed.) (1971), Les théories de la causalité, París: Presses Universitaires de France, pp. 112-132.

- (1968k), "Physical Time: The Objective and Relational Theory", Philosophy of Science 35: 355-388.

- (19681), “La verification des theories scientifiques”, en Démonstration, Vérification, Justification: Entretiens de l'Institut International de Philosophie, Lovaina-París: Nauwelaerts, pp. 145-159. Comentarios de N. Rescher, N. Rothenstreich, G. Hirsch, G. Granger, A. J. Ayer, J. Hersch, J. Hyppolite, A. G. M van Melsen y H. L. van Breda y respuesta del autor: pp. 160-179.

- (1968m), "Les concepts de modèle”, L’Âge de la Science 1: 165-180.

- (1968n), "Theory of Partial Truth: Not Proved Inconsistent", Philosophy and Phenomenological Research 29(2): 297298.

- (1969a), "Corrections to Foundations of Physics: Correct and Incorrect”, Synthèse 19: 443-452.

- (1969b), “The Metaphysics, Epistemology and Methodology of Levels”, en L. L. Whyte, A. G. Wilson y D. Wilson (eds.), Hierarchical Levels, Nueva York: American Elsevier, pp. 17-28.

- (1969c), La investigación científica, Barcelona: Ariel. Edición revisada, México D.F.: Siglo XXI, 2000.

- (1969d), "Alexander von Humboldt und die Philosophie”, en Pfeiffer, H. (ed.), Alexander von Humboldt, Munich: Piper and Co., pp. 17-30. Existe traducción al castellano: "Alexander von Humboldt y la filosofía”, Folia Humanística (Barcelona) 8: 535-546.

- (1969e), "What are Physical Theories About?”, American Philosophical Quarterly, Supplementary Monograph No. 3.

- (1969f), “Analogy, Simulation, Representation”, Revue International de Philosophie 23: 16-33.

- (1969e), "Mach’s Beitrag zur Grundlegung der Mechanik”, Philosophia Naturalis 11: 189-213.

- (1969f), "Four Models of Human Migration: An Exercise in Mathematical Sociology, Archiv für Rechts- und Sozialphilosophie 55: 451-462.

- (1969g), "Models in Theoretical Science", Proceedings of the XIV ${ }^{\text {th }}$ International Congress of Philosophy III, Viena: Herder, pp. 208-217.

- y Kálnay, A. J. (1969), “A Covariant Position Operator for The Relativistic Electron”, Progress of Theoretical Physics 42(6): 1.445-1.459.

- (1970a), "Problems Concerning Inter-theory Relations”, en Weingartner, P. y G. Zecha (eds.), Induction, Physics and Ethics, Dordrecht: Reidel, pp. 285-315.

- (1970b), "The Arrow of Time", International Journal of Theoretical Physics 3: 77-78.

- (1970c), “Time Asymmetry, Time Reversal and Irreversibility, Studium Generale 23: 562-570. Reproducido en Fraser, J.T. y otros (eds.), The Study of Time, Berlín-Heidelberg-Nueva York: Springer, pp. 122-130.

- (1970jd, "Virtual Processes and Virtual Particles: Real or Fictitious?", International Journal of Theoretical Physics 3: 507-508.

- (1971a), "Is Scientific Metaphysics Possible?", Journal of Philosophy 68: 507-520. Existe traducción al castellano: “Es posible una metafísica científica?”, Teorema 3: 435-454.

- (1971b), “A Philosophical Obstacle to the Rise of New Theories in Microphysics”, en Bastin, E.W. (ed.), Quantum theory and Beyond, Cambridge: Cambridge University Press, pp. 263-273.

- (1971c), “A Mathematical Theory of the Dimensions And Units of Physical Quantities”, en Bunge, M. (ed.), Problems in the Foundations of Physics, Berlín-Heidelberg: Springer, pp. 1-16.

_ (1971d), “On Method in the Philosophy of Science”, Archives de Philosophie 34: 551-574.

- (1971e), "Space and Time in Modern Science”, II Bienal de Ciência e Humanismo, San Pablo: Fundação Bienal de São Paulo, pp. 21-34. 
- (1972a), "Modelo del dilema electoral argentino", Ciencia Nueva 21: 52-54.

- (1972b), "Seudociencia y seudo filosofía: dos monólogos paralelos, Ciencia Nueva 15: 41-43.

- (1972c), "A Program for the Semantics of Science", Journal of Philosophical Logic 1: 317-328.

- (1973a), Philosophy of Physics, Dordrecht: Reidel.

- (1973b), Method, Matter and Model, Dordrecht: Reidel.

- (1973c), "A Decision Theoretic Model of The American War in Vietnam", Theory and Decision 3: 328-338. Existe traducción al alemán: "Enscheidungstheoretische Modelle in der Politik: Vietnam", en Schaeffer, R.S. y W.C. Zimmerli (eds.), Wissenschaftstheorie der Geisteswissenschaften, Hamburgo: Hoffmann \& Campe, pp. 309-323.

- (1973d), The Methodology Unit of Science, Boston: Reidel (ed.).

- (1973e), "Meaning in Science", Proceedings of the XV ${ }^{\text {th }}$ World Congress of Philosophy II, Sofía: Sofia Press, pp. 281286. Existe traducción al castellano: "El significado en ciencia”, Teoría (México) 1(1): 65-76.

- (1973f), "The Role of Forecasting in Planning", Theory and Decision 3: 207-221.

- (1974a), Philosophie de la physique, París: Seuil.

- (1974b), "The Concept of Social Structure”, en Leinfellner, W. y W. Köhler (eds.), Developments in the Methodology of Social Science, Dordrecht/Boston: Reidel, pp. 175-215.

- (1974c), Treatise on Basic Philosophy, vol. 1, Sense and Reference, Dordrecht/Boston: Reidel.

- (1974d), Treatise on Basic Philosophy, vol. 2, Interpretation and Truth, Dordrecht/Boston: Reidel.

- (1974e), The Methodology of Development Indicators, París: Methods and Analysis Division, Department of Social Sciences, UNESCO.

- (1975a), “Crítica de la noción fregeana de predicado”, Revista Latinoamericana de Filosofia 1: 5-8.

- (1975b), “'Hay proposiciones?”, en Aspectos de la filosofia de W.V. Quine, Valencia: Teorema, pp. 53-68.

- (1975c), "A Critical Examination of Dialectics", en Perelman, Ch. (ed.), Dialectics/Dialectique, Nijhoff: La Haya, pp. 66-77.

- (1975d), "What is a Quality of Life Indicator?", Social Indicators Research 2: 65-80.

- y A. J. Kálnay (1975). "Welches sind die Besonderheiten der Quantenphysic gegenuiber der klassischen Physik?", en Haller, R. y J Götschi, (eds.), Philosophie und Physik, Braunschweig: Vieweg, pp. 25-38. Existe traducción al francés en Fundamenta Scientiae 11 (1976).

- (1976a), "The Relevance of Philosophy to Social Science", en Shea, W. (ed.), Basic Issues in the Philosophy of Science, Nueva York: Neale Watson, pp. 136-155. Existe versión en castellano: La filosofía y las ciencias sociales, México D.F.: Grijalbo, pp. 43-69.

- (1976b), "El método en la biología”, Naturaleza (México) 7: 70-81.

- (1976c), "A Model for Processes Combining Competition with Cooperation”, Mathematical Modelling 1: 21-23.

- (1976d), "Possibility and Probability", en Harper, W. y C. Hooker (eds.), Foundations of Probability Theory, Statistical Inference and Statistical Theories of Science, Vol. III, Dordrecht-Boston: Reidel, pp. 17-33.

- (1976e), "A Model for Processes Combining Competition with Cooperation", Applied Mathematical Modelling 1: 21-23. Existe traducción al castellano: "Modelos para procesos que combinan competencia y cooperación", Revista Iberoamericana de Autogestión y Acción Comunal 6(13/14) (1988): 27-32.

- (1976f), "Is Science Value-Free and Morally Neutral?", Philosophy and Social Action 2(4): 5-18.

- y M. García Sucre (1976). "Differentiation, Participation and Cohesion”, Quality and Quantity 10: 171-178.

- y A. García Máynez (1976), "A Relational Theory of Physical Space", International Journal of Theoretical Physics 15(12): 961-972.

- (1977a), "Emergence and the Mind", Neuroscience 2: 501-508.

- (1977b), "Levels and Reduction", American Journal of Physiology: Regulatory, Integrative and Comparative Physiology 2: 75-82. 
- (1977c), Treatise on Basic Philosophy, vol. 3, The Furniture of the World, Dordrecht-Boston: Reidel.

- (1977d), “A Systems Concept of the International System”, en Bunge, M., Galtung, J. y M. Malitza (eds.), Mathematical Approaches to International Relations, Bucharest: Romanian Academy of Social and Political Sciences, pp. 291-305.

- y A. Sangalli (1977), “A Theory of Properties and Kinds”, International Journal of General Systems 3: 183-190.

- y A. García Máynez (1977), “A Relational Theory of Physical Space”, International Journal of Theoretical Physics 15: 961-972.

- (1978a), "Review of W. Stegmüller's The Structure and Dynamics of Theories (1976)", Mathematical Reviews 55 (2): 333 (\#2480).

- (1978b), “A Model of Evolution”, Applied Mathematical Modelling 2: 201-204.

- (1978c), "Iatrofilosofía”, en Alonso de Florida, F. (comp.), Ensayos de Yatrofilosofía, México: Academia Nacional de Medicina, pp. 3-5.

- (1978d), "Physical Space”, en Svilar, M. y A. Mercier (eds.), Space, Berna-Frankfurt-Las Vegas: Peter Lang, pp. 133. 148.

- y R. Llinás (1978a), "Restricted Applicability of the Concept of Command Neuron in Neuroscience: Dangers of Metaphors", Behavior and Brain Science 1: 30-31.

- y - (1978b), "The Mind-Body Problem in the Light of Contemporary Biology", $16^{\text {th }}$ World Congress of Philosophy: Section Papers, Frankfurt am Main: Peter Lang, pp. 131-133.

- (1979a), “A Systems Concept of Society: Beyond Individualism and Holism”, Theory and Decision 10: 13-30.

- (1979b), “The Mind-Body Problem in an Evolutionary Perspective”, en Wolstenhome, G. y M. O’Connor (eds.), Brain and Mind, Ciba Foundation Series 69, Amsterdam: Excerpta Medica, pp. 53-63.

- (1979c), Treatise on Basic Philosophy, vol. 4, A World of Systems, Dordrecht-Boston, Reidel.

- (1979d), "The Mind-Body Problem, Information theory, and Christian Dogma”, Neuroscience 4: 453-454.

- (1979e). "La bancarrota del dualismo psiconeural”, en Fernández Guardiola, A. (comp.), La conciencia, México: Trillas.

- (1979f), "Some Topical Problems in Biophilosophy”, Journal of Social and Biological Structures 2: 155-172.

- (1979g), "The Five Buds of Technophilosophy", Technology in Society 1: 67-74.

- (1979h), "Philosophical Inputs and Outputs of Technology”, en Bugiarello, G. y D.B. Donner (eds.), The History and Philosophy of Technology, Urbana: University of Illinois Press, pp. 262-281.

- (1979j), “El finalismo en biología, psicología y sociología”, Revista Latinoamericana de Filosofía 5: 33-40.

- (1979k), “Ideologizar la ciencia o cientificizar la ideología?”, en Otero, M. (ed.), Ideología y Ciencias Sociales, México: Universidad Nacional Autónoma de México, pp. 41-51.

- (1980a), The Mind-Body Problem, Oxford: Pergamon. Existe versión en castellano: El problema mente-cerebro: Un enfoque psicológico, Madrid: Tecnos, 1985.

- (1980b), Ciencia y desarrollo, Buenos Aires: Siglo Veinte.

- (1980c), Materialismo y ciencia, Barcelona: Ariel. Reedición corregida y ampliada, Pamplona: Laetoli, 2013.

- (1980d), “Introduction”, en Bindra, D. (ed.), The Brain's Mind, Nueva York: Gardner Press, pp. 1-5.

- (1980e), “Technoethics”, en Kranzberg, M. (ed.), Ethics in an Age of Pervasive Technology, Boulder, CO: Westview Press, pp. 139-142.

- (1980f), "La función de la ciencia básica en el desarrollo nacional”, Tecnología y Desarrollo 4: 153-170.

- (1980g), “The Psyconeural Identity Theory”, en Bindra, D. (ed.), The Brain's Mind, Nueva York: Gardner Press, pp. 89-108. Existe traducción al castellano: "Una teoría materialista de la mente", Episteme 2(4): 43-50.

- (1981a), "From Mindless Neuroscience and Brainless Psychology to Neuropsychology", en Madsen, K.B. y L.P. Mos (eds.) (1985), Annals of Theoretical Psychology 3, Nueva York: Plenum Press, 115-133. Comentarios de M.C. Corballis y P.C. Dodwell. Existe traducción al castellano: "Desde una neurociencia sin mente y una psicología sin 
cerebro a una neuropsicología", Revista de Filosofía (Chile) 20 (1982):5-22.

- (1981b), "Half Truths", en Morscher, E., Neumaier, O. y G. Zecha (eds.), Philosophie als Wissenschaft, Bad Reichenhall: Comes Verlag, pp. 87-91.

- (1981c), “Development Indicators”, Social Indicators Research 9: 369-385. Versión revisada de Bunge (1974e).

- (1981d), Teoría y realidad, Barcelona: Ariel (3º edición).

- (1981e), Scientific Materialism, Dordrecht: Reidel.

- (1981f), "Four Concepts of Probability", Applied Mathematical Modelling 5: 306-312.

- (1981g), "Biopopulations, not Biospecies, are Individuals and Evolve", Behavioral and Brain Science 4: 284-285.

- (1981h), "Las funciones de la ciencia y de la técnica en el desarrollo nacional", en Memoria del Primer Seminario Nacional sobre Politica de Desarrollo Científico y Tecnológico, Vol. 1, Quito: Editorial Voluntad, pp. 115-138.

- (1981i), "Ciencia básica, ciencia aplicada, técnica y producción. Diferencias y relaciones", en Memoria del Primer Seminario Nacional sobre Politica de Desarrollo Cientifico y Tecnológico, Vol. 2, Quito: Editorial Voluntad, pp. 51-68. Reproducido en El Pais (Madrid) 18 y 20 de junio de 1982.

- (1982a), Economía y filosofía, Madrid: Tecnos.

- (1982b), "Is Chemistry a Branch of Physics?", Zeitschrift für allgemeine Wissenschaftstheorie 13: 209-223.

- (1983a), Lingiística y filosofía, Barcelona: Ariel.

- (1983b), "Speculation: Wild and Sound", New Ideas in Psychology 1: 3-6.

- (1983c), Treatise on Basic Philosophy, vol.5, Exploring the World, Dordrecht: Reidel.

- (1983d), Treatise on Basic Philosophy, vol. 6, Understanding the World, Dordrecht: Reidel

- (1983e), "La necesidad de mantener la dicotomía entre verdades de razón y verdades de hecho", Revista Latinoamericana de Filosofía 10: 63-69.

- y A.J. Kálnay (1983a). "Solution to two Paradoxes in the Quantum Theory of Unstable Systems", Nuovo Cimento B77: $1-9$.

- y - (1983b). "Real Successive Measurements on Unstable Quantum Systems take Nonvanishing Time Intervals and do not Prevent them from Decaying", Nuovo Cimento B77: 10-18.

- (1984a), "Philosophical Problems in Linguistics", Erkenntnis 21: 107-173.

- (1984b), "What is Pseudoscience?", The Skeptical Inquirer 9(1): 36-46.

- (1985a), Treatise on Basic Philosophy, vol. 7, part II, Life Science, Social Science, and Technology, Dordrecht: Reidel.

- (1985b), Seudociencia e ideología, Madrid: Alianza.

- (1986a), "Science, Technology and Ideology in the Hispanic world", Free Inquiry 6(3): 36-49. Existe traducción al castellano: "Ciencia e ideología en el mundo hispánico", Interciencia 11: 120-125.

- (1986b), "A Philosopher Looks at the Current Debate on Language Acquisition”, en Gopnik, I. y M. Gopnik (eds.), From Models to Modules, Norwood, NJ: AblexPubls. Co., pp. 229-239.

- (1987), "Two Controversies in Evolutionary biology: Saltationism and Cladism”, en Rescher, N. (ed.), Scientific Inquiry in Philosophical Perspective, Lanham, MD: University Press of America, pp. 129-145.

- (1988a), Filosofía de la psicología, Barcelona: Ariel.

- (1988b), "Why Parapsychology cannot Become a Science", Behavioral and Brain Sciences 10: 576-577.

- (1988c), "Basic Science is Innocent, Applied Science and Technology can be Guilty", en Lemarchand, G.E. y A.R. Pedace (eds.), Scientists, Peace and Disarmament, Singapur: World Scientific, pp. 245-261.

- (1989a), "Reduktion und Integration, Systeme und Niveaus, Monismus und Dualismus", en Pöppel, E. (ed.), Gehirn und Bewusstsein, Weinheim: VCH, pp. 87-104.

- (1989b), "From Neuron to Mind", News in Physiological Sciences 4: 206-209.

- (1989c), Mente y sociedad, Madrid: Alianza. 
- (1989d), "Game Theory is not a Useful Tool for Political Science”, Epistemología 12: 195-212.

- (1989e), Treatise on Basic Philosophy, vol. 8, The Good and the Right, Dordrecht-Boston, Reidel.

- (1989f), "The Popular Perception of Science in North America”, Transactions of the Royal Society of Canada Ser. V. Vol. V, 269-289. Existe traducción al castellano: "La percepción popular de la ciencia en Norteamérica", El Ojo Escéptico 1(2) (1991): 1-4.

- (1990), "What Kind of Discipline is Psychology?”, New Ideas in Psychology 8: 121-137.

- (1991a), “A Philosophical Perspective on the Mind-Body Problem”, Proceedings of the American Philosophical Society 135: 513-523.

_ (1991b), “A Skeptic’s Beliefs and Disbeliefs”, New Ideas in Psychology 9: 131-149.

- (1991c), “Una caricatura de la ciencia: La novísima sociología de la ciencia”, Interciencia 16: 69-77.

- (1991d), "A Critical Examination of the New Sociology of Science”, $1^{\text {st }}$ part, Philosophy of the Social Sciences 21: 524-560.

- (1991e), "El sistema técnica-ciencia-filosofía: un triángulo fértil”, Telos 24:13-22. Existe traducción al francés: "Le système technique-science-philosophie: un ménage à trois feconde”, Revue Internationale de Systémique 5: 171-180.

- (1992a), "A Critical Examination of the New Sociology of Science", $2^{\text {nd }}$ part, Philosophy of the Social Sciences 22: 4676.

- (1992i), "Sette paradigmi cosmologici: L'animale, la scala, il fiume, la nuvola, la macchina, il libro e il sistema dei sistema", Aquinas 35: 219-235. La versión original se publicó después como: "Seven Cosmological Paradigms: Animal, Ladder, River, Cloud, Machine, Book, and System of Systems”, en Sánchez Sorondo, M. (ed.), Physica, Cosmologia, Naturphilosophie: Nuovi Approcci, Roma: Herder-Università Lateranense, pp. 115-131, 1993.

- (1992/1993), “Eine Kritik der Grundlagen der rationalen Wahl”, Zeitschrift für Wissenschaftliche Forschung 7/9: 19. 33.

- (1993a). "Die Bedeutung der Philosophie für die Psychologie”, en Montada, L. (ed.), Bericht über den 38. Kongress der Deutschen Gesellschaft für Psychologie in Trier 1992, Gotinga: Hogrefe, vol. 22, pp. 51-63.

- (1993b), Filosofía de la ciencia, Buenos Aires: Siglo Veinte.

- (1993c), "Realism and Antirealism in Social Science", Theory and Decision 35: 207-235.

- (1993d), Sociología de la ciencia, Buenos Aires: Siglo Veinte.

- (1994a), "L'écart entre les mathématiques et le reel”, en Porte, M. (ed.), Passion des forms, Fontenay-St Cloud: E.N.S. Editions, pp. 165-173.

- (1994b), "Quality, Quantity, Pseudoquantity and Measurement in Social Science", Journal of Quantitative Linguistics 2: 1-10.

- (1994c), “Counter-Enlightenment in Contemporary Social Studies”, en Kurtz, P. y T.J. Madigan (eds.), Challenges to Enlightenment. In Defense of Reason and Science, Búfalo: Prometheus, pp. 25-42.

- (1996a), "In Praise of Intolerance to Charlatanism in Academia”, Annals of the New York Academy of Sciences 778 : 96-116.

- (1996b), Finding Philosophy in Social Sciences, New Haven: Yale University Press.

- (1996c), “The Seven Pillars of Poppers's Social Philosophy”, Philosophy of the Social Sciences 26: 528-556.

- y M. Mahner (1996a), “Is Religious Education Compatible with Science Education?”, Science Ë Education 5: 96. 116.

- y - (1996b), "The Incompatibility of Science and Religion Sustained: a Reply to Our Critics”, Science $\mathcal{E}$ Education 5: 189-199.

- (1997a), "Moderate Mathematical Fictionism”, en Agazzi, E. y G. Darwas (eds.), Philosophy of Mathematics Today, Dordrecht-Boston: Kluwer Academic, pp. 51-71.

- (1997b), Epistemología, México D.F.: Siglo XXI.

- (1997c), “A New Look at Moral Realism”, en Garzón Valdés, E., Krawietz, W., von Wright, G.H. y R. Zimmerling 
(eds.), Normative Systems in Legal and Moral Theory, Berlín: Dunckler \& Humblot, pp. 17-26.

- (1997d), "Mechanism and Explanation", Philosophy of the Social Sciences 27: 410-465.

- y M. Mahner (1997), Foundations of Biophilosophy, Berlín-Heidelberg-Nueva York: Springer.

- (1998a), Sociología de la ciencia, Buenos Aires: Sudamericana.

- (1998b), Social Science under Debate, Toronto: University of Toronto Press.

- y L. Marone (1998), "La explicación en ecología”, Boletin de la Asociación Argentina de Ecologia 7(2): 35-37.

- (1999a), Buscar la filosofía en la ciencia social, México D.F.: Siglo XXI.

- (1999b), Las ciencias sociales en discusión, Buenos Aires: Sudamericana.

- (2000a), "Ten Modes of Individualism - None of which Works - and their Alternatives", Philosophy of the Social Sciences 30: 384-406.

- (2000b), "Systemism: The Alternative to Individualism and Holism", Journal of Socio-Economics 29: 147-157.

- (2000c), La relación entre la sociología y la filosofia, Madrid: Edaf.

- (2000d), “Absolute Skepticism equals Dogmatism”, Free Inquiry 24(4): 34-36.

- (2002a), "Quantons are Quaint but Basic and Real”, Science E Education 12: 587-597.

- (2002b), Crisis y reconstrucción de la filosofía, Barcelona-Buenos Aires: Gedisa.

- (2003a), "Interpretation and Hypothesis in Social Studies", en Boudon, R., Cherkaoui, M. y R. Demeulenaere (eds.), The European Tradition in Qualitative Research, vol. IV, Londres: Sage Publications, pp. 20-40.

- (2003b), "Velocity Operators and Time Energy Relations in Relativistic Quantum Mechanics", International Journal of Theoretical Physics 42: 135-142.

- (2003c), "Twenty-Five Centuries of Quantum Physics: From Pythagoras to Us, and from Subjectivism to Realism", Science E⿱ Education 12: 445-466.

- (2003d), Cápsulas, Barcelona: Gedisa.

_ , Thurler, G. y otros (2003), "Toward a Systemic Approach to Disease", ComPlexUs 1: 117-122.

- (2004a), Emergence and Convergence, Toronto: University of Toronto Press.

- (2004b), "How Does it Work? The Search for Explanatory Mechanisms", Philosophy of the Social Sciences 34: 182. 210.

- (2004c). "Clarifying some Misunderstandings about Social Systems and their Mechanisms", Philosophy of the Social Sciences 34: 371-381.

- y Mahner, M. (2004), Über die Natur der Dinge, Sttutgart: S. Hirzel.

- (2006), "A Systemic Perspective on Crime”, en Wikström, P.-O. y R. Sampson (eds.), The Explanation of Crime, Cambridge: Cambridge University Press, pp. 8-30.

- (2007a), "Max Weber Did not Practice the Philosophy He Preached", en McFalls, L. (ed.), Max Weber's "Objectivity" Revisited, Toronto: University of Toronto Press, pp. 119-134.

- (2007b), Una filosofía realista para el nuevo milenio, Lima: Universidad Garcilaso de la Vega, 2a edición.

- (2007c), A la caza de la realidad, Barcelona-Buenos Aires: Gedisa.

- (2008a), Le materialisme scientifique, París: Syllepse.

- (2008b), Tratado de filosofía, vol 1, Semántica I. Sentido y referencia, Barcelona-Buenos Aires: Gedisa.

- (2009a), Filosofía política, Barcelona/Buenos Aires: Gedisa.

- (2009b), Tratado de filosofía, vol. 2, Semántica II. Interpretación y verdad, Barcelona-Buenos Aires: Gedisa.

- (2010a), Matter and Mind, Dordrecht-Heidelberg-Londres-Nueva York: Springer.

- (2010b), Las pseudociencias ivaya timo!, Pamplona: Laetoli.

- (2011), Tratado de filosofía, vol. 3, Ontología I. El moblaje del mundo, Barcelona-Buenos Aires, Gedisa. 
16 | Gustavo E. Romero y Pablo M. Jacovkis

- (2012a), Evaluating Philosophies, Dordrecht-Heidelberg-Londres-Nueva York: Springer-Verlag.

- (2012b), Filosofía para médicos, Barcelona-Buenos Aires: Gedisa.

- (2012c), Tratado de filosofía, vol 4, Ontología II, Un mundo de sistemas, Barcelona-Buenos Aires: Gedisa.

- (2013a), Medical Philosophy, Singapur: World Scientific.

- (2013b), "Bruce Trigger and the Philosophical Matrix of Scientific Research”, en Chrisomalis, S. y A. Costopoulos (eds.), Human Expeditions Inspired by Bruce Trigger, Toronto: Toronto University Press, pp. 143-159.

- y C. Gabetta (comps.) (2013), ¿Tiene porvenir el socialismo?, Buenos Aires: Eudeba.

- (2014a), “In Defense of Scientism”, Free Inquiry 35(1): 24-28.

- (2014b), Entre dos mundos, Buenos Aires: EUDEBA.

- (2015), Evaluando filosofías, Barcelona-Buenos Aires: Gedisa.

- (2016), Between Two Worlds, Berlín-Heidelberg-Nueva York: Springer. Traducción -con modificaciones- de Bunge (2014b). 